\title{
Epithelioid angiosarcoma of the small intestine presenting with intractable gastrointestinal bleeding: An uncommon tumor with diagnostic challenge and poor prognosis
}

\author{
Chinelo Pamela Onyenekwu, Reena Singh, James Adam Miller, Mohamed Mostafa* \\ Department of Pathology, Medical College of Wisconsin, Milwaukee, WI, USA
}

Received: April 21, 2021

DOI: $10.5430 /$ crcp.v8n1p17
Accepted: June 17, 2021

Online Published: July 1, 2021

URL: https://doi.org/10.5430/crcp.v8n1p17

\begin{abstract}
Introduction: Angiosarcoma of the gastrointestinal tract is a rare, aggressive malignant neoplasm of vascular origin. Commonly, this tumor occurs due to metastases from other sites like the head and neck region and the soft tissue. When occurring as a primary tumor of the intestines, this entity poses a diagnostic dilemma due to its non-specific clinical presentation, coupled to its infiltrative features that may occur without an obvious lesion amenable to routine gastrointestinal interventions. The findings from an autopsy and surgical case with a brief review of its diagnostic features and relevant literature are discussed.

Methods: We present a 73-year-old male with a history of a recent onset of gastrointestinal bleeding who was referred to our facility for persisting bleeding. He underwent endoscopy without an identifiable source of bleeding and subsequently received a colectomy at our facility, with repeated blood transfusions for persisting gastrointestinal bleeding. He was eventually transitioned to hospice and died shortly after presentation. A complete autopsy was performed.

Results: There was anasarca with hemoperitoneum and intestinal intraluminal blood. Present in the small intestinal mucosa were three hemorrhagic nodules. Histologically, the nodules demonstrated medium to large epithelioid vasoformative neoplastic cells infiltrating through the wall of the small intestine and extending to the omentum. The findings from the gross and histology examination, as well as the immunohistochemical stains were consistent with an epithelioid angiosarcoma.

Conclusion: Primary epithelioid angiosarcoma of the intestine is an uncommon aggressive tumor that poses a diagnostic challenge. Although rare, this diagnosis needs to be considered in patients with intractable gastrointestinal bleeding. Due to its morphologic overlap with other benign and malignant neoplasms, the use of ancillary testing during histologic examination is key in arriving at an accurate diagnosis.
\end{abstract}

Key Words: Angiosarcoma, Bleeding, Epithelioid, Gastrointestinal, Malignant neoplasm

\section{INTRODUCTION}

Approximately three-quarters of the gastrointestinal tract is comprised of the small bowel, but primary small intestinal malignant neoplasms are unusual. Of the mesenchy- mal tumors, gastrointestinal stromal tumors are the most common. ${ }^{[1]}$ Angiosarcoma (AS) is a malignant vasoproliferative lesion that constitutes about 1 to 2 of every 100 sarcoma cases, and the most common primary sites include

*Correspondence: Mohamed Mostafa; Email: mmostafa@mcw.edu; Address: 9200 W Wisconsin Avenue, Milwaukee, WI 53226, USA 
skin and subcutis. ${ }^{[2,3]}$ About half of the AS cases involving the gastrointestinal tract are metastases from extra-intestinal sites. ${ }^{[4,5]}$ The epithelioid variant of intestinal AS poses a diagnostic challenge due both to its rarity and mimicry of epithelial malignancies. ${ }^{[4]}$ Herein, we report a case of a 73-year-old male who presented with persistent gastrointestinal bleeding warranting surgery, deteriorated rapidly postsurgery, and was diagnosed post-mortem with epithelioid AS. The clinical features, associated factors, histologic and immunochemical features, and the differential diagnosis of epithelioid AS are discussed.

\section{Case presentation}

A 73-year-old man with a history of abdominal aortic aneurysm and endovascular aneurysmal repair two years prior to presentation was admitted to the medical intensive care unit for persisting gastrointestinal bleeding as a transfer from an outside hospital. He underwent a right hemicolectomy at the outside institution for ongoing gastrointestinal bleeding, which required continuous transfusions to mitigate hemorrhagic shock. Esophagogastroduodenoscopy and colonoscopy were performed at our hospital, and the latter demonstrated punctate hemorrhages, multiple slightly raised tan-red lesions, and small ulcers in the terminal ileum (see Figure 1A). A subsequent capsule endoscopy revealed small angioectasia in the proximal small bowel and friable mucosal erosion with fresh blood in the distal small bowel just proximal to the previous surgical anastomotic site (see Figure 1B).
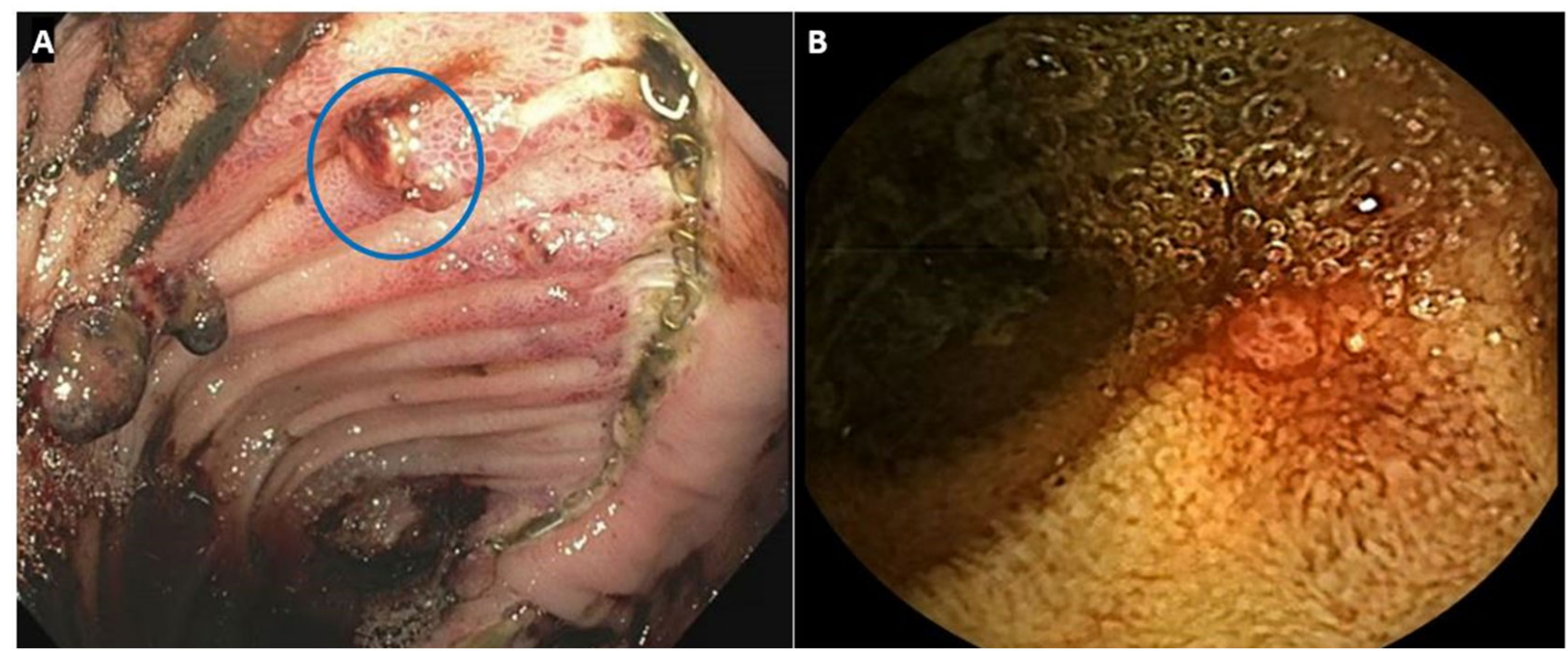

Figure 1. (A) Colonoscopy image showing old hemorrhage and blood clot as well as a tan-red slightly raised lesion with small ulcer (blue circle) in the ileal side of the ileocolonic anastomosis. (B) Capsule endoscopy demonstrates friable mucosal erosion in the distal small bowel

Following capsule endoscopy, the patient was taken for exploratory laparotomy and found to have a massive hemoperitoneum due to active bleeding from the anastomotic site. The previous site was excised, and an ileocolonic anastomosis was created.

He continued to bleed, and another exploratory laparotomy was performed and resulted in total colectomy and end ileostomy when an obvious bleeding source could not be identified. Nonetheless, his gastrointestinal bleeding persisted, and he required multiple units of red blood cells daily. The decision was made to transition to comfort care. The patient died a few hours later. Consent for a complete autopsy was obtained from the spouse.

\section{Pathology examination}

Autopsy demonstrated anasarca, 3.2 liters hemoperitoneum, and 2.0 liters of intestinal intraluminal blood. Three hemorrhagic nodules in the small intestinal mucosa and serosa ranging from 0.3 to $0.9 \mathrm{~cm}$ were identified (see Figure 2).

Rapid formalin-fixed paraffin-embedded sections (4 $\mu \mathrm{m})$, stained with hematoxylin-eosin, from the autopsy showed the nodular lesions to be an epithelioid, vasoformative malignancy. The neoplastic cells were medium to large with irregular nuclear contour, open chromatin, prominent eosinophilic nucleoli, and a moderate amount of cytoplasm. Scattered mitotic figures, including atypical forms, were also present (see Figure 3A). The small bowel wall showed extensive infiltration of the neoplastic cells. 


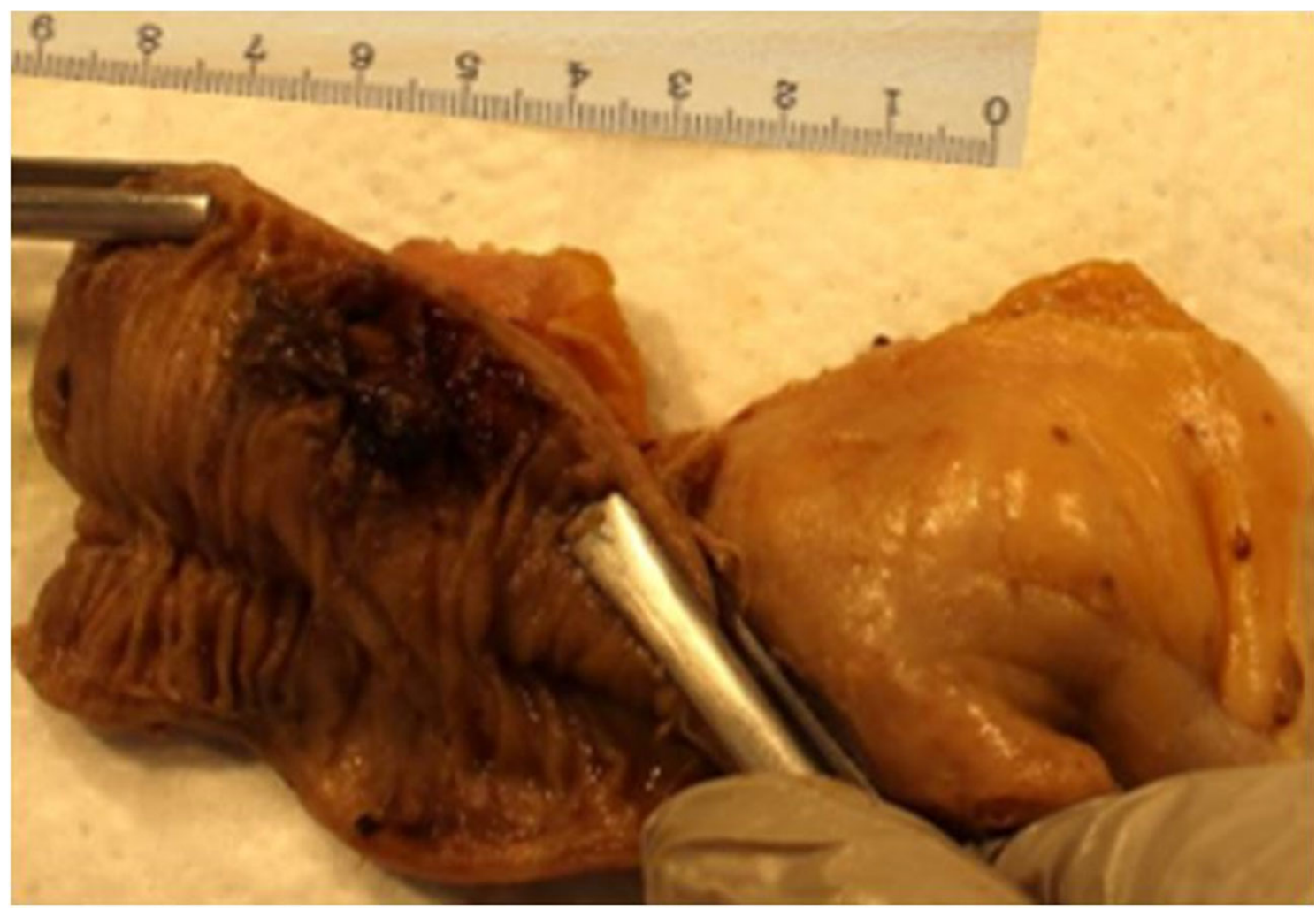

Figure 2. Gross photo from the autopsy specimen demonstrates hemorrhagic nodular lesions on the small bowel mucosa

Gross examination of the surgical resection specimens showed small bowel with purple-pink serosa and folded tanred mucosa, with a small nodular lesion. The colon exhibited a folded tan-pink mucosa with numerous intact diverticula and tan-brown lesions ranging from $0.2-0.8 \mathrm{~cm}$. Histological examination of the surgical resection specimen, which was performed after evaluating the rapid autopsy sections, demonstrated an involvement of the small intestine, colon, and omentum by the same malignant process. Immunohistochemical stains revealed the neoplastic cells to be strongly positive for CD31 and ERG while negative for CD45 and HHV-8 (see Figure 3B-F). In-situ hybridization for EBV was negative. The morphology and the immunophenotypic profile were in keeping with an epithelioid AS. The disease was diffusely multifocal within the gastrointestinal tract, but a thorough histologic examination of the aortic aneurysm graft site and other organs from the autopsy did not reveal any malignancy.

\section{Discussion}

Angiosarcomas involving the deep soft tissues and gastrointestinal tract are typically aggressive with a dismal outcome. ${ }^{[6]}$ Limited number of case reports of primary AS involving the gastrointestinal tract have been documented in the literature, with a small proportion of these cases describing tumors of small bowel origin. ${ }^{[6-10]}$ AS has a wide Published by Sciedu Press range of histologic features and may present as a neoplasm with poorly differentiated, high-grade spindled or epithelioid cells or a well-differentiated tumor with well-formed vascular channels. Commonly involved sites include the head and neck skin in the older population and the breast, usually in association with radiation therapy for breast cancer. ${ }^{[1]}$ AS has also been reported to occur at sites of or adjacent to endovascular and bypass grafts. ${ }^{[12,13]}$ There is an increased incidence in those with prior radiation therapy, appreciable vinyl chloride or arsenic exposure, or prolonged lymphedema, usually as a sequela to axillary lymph node dissection for breast cancer. ${ }^{[3,14,15]}$

Our patient had an aortic graft and axillary-femoral bypass however the possibility of this location as a primary site was excluded by performing a thorough gross and microscopic examination. Additionally, there was no history of radiation therapy, chronic lymphedema, or industrial chemical exposure. Clinically, AS in the skin and soft tissue usually presents with erythematous, multifocal, and painless nodules with indistinct borders. The lesions tend to recur and have high metastatic potential. Epithelioid AS is even more aggressive than its conventional counterpart and in the small intestine can present with non-specific signs and symptoms, including abdominal pain, persistent/recurrent gastrointestinal bleeding, anemia, weight loss, and bowel perforation. ${ }^{[3]}$ 
These variable presentations make rapid and accurate diag- the lesion complicates complete surgical removal. ${ }^{[3,5]}$ nosis challenging. The infiltrative and aggressive nature of
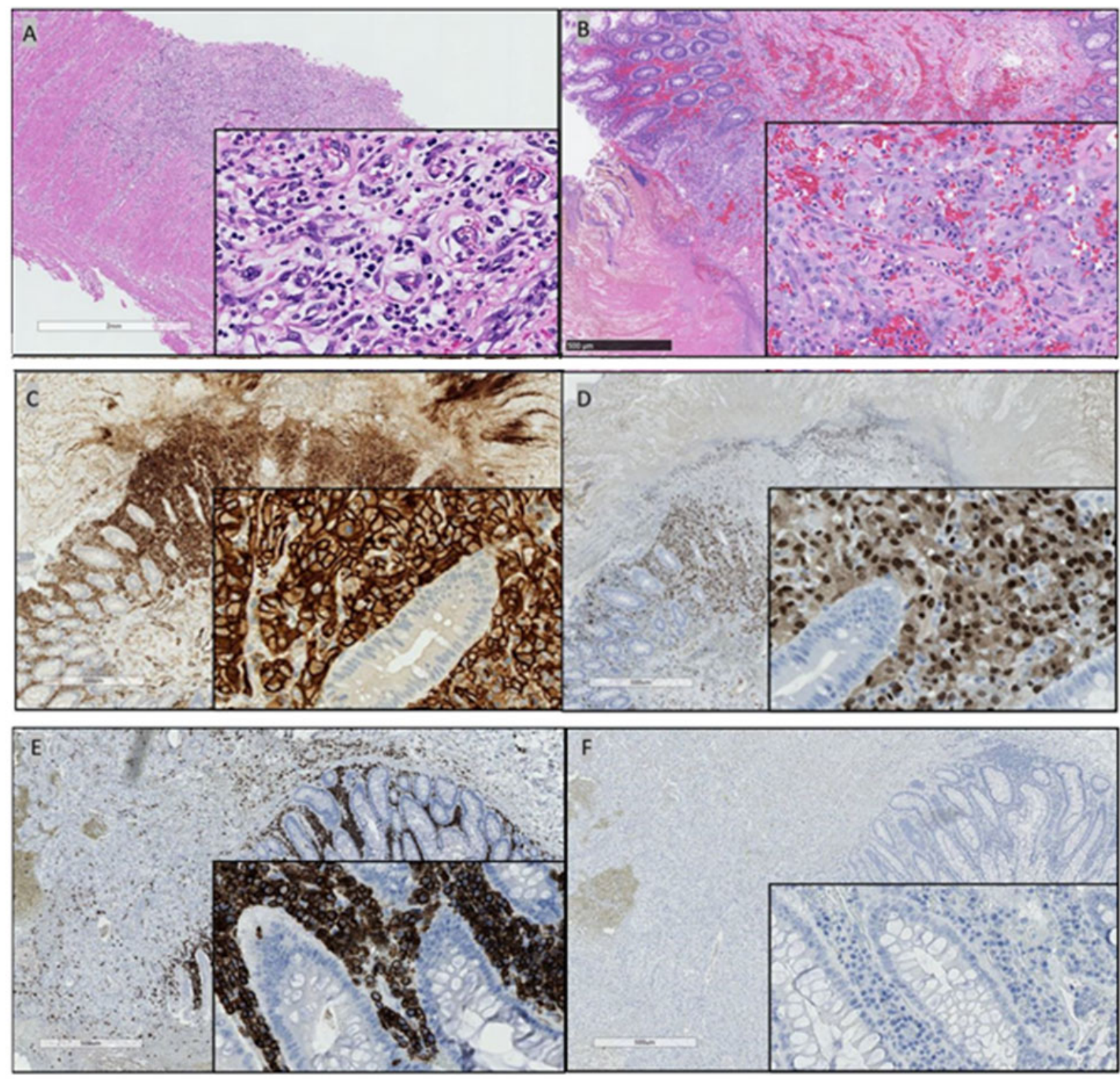

Figure 3. Histology sections from the autopsy specimen (3A) and the surgical resection specimen (3B to 3F). 3A: Hematoxylin \& eosin, low power view of small bowel on autopsy specimen showing a lesion infiltrating through the bowel, inset shows higher power view of lesion; 3B: Hematoxylin and eosin, low power view (x4) of surgical specimen with inset of tumor at higher power; 3C: Immunohistochemical stain for CD31 shown at $\mathrm{x} 4$ and $\mathrm{x} 20$ (inset); 3D: Same specimen in 2B showing immunohistochemical stain for ERG at x4 and x20 (inset) 3E: Immunohistochemical stain for CD45 shown at x4 and $\mathrm{x} 20$ (inset); 3F. Same specimen in 2B showing immunohistochemical stain for HHV8 at x4 and x20 (inset).

Definitive diagnosis is based on pathological examination. Grossly, there may be hemorrhagic nodules with indefinite borders and ulceration. Endoscopic biopsy of the lesions may be helpful; however, this may only be feasible in a few cases when the lesion can be visualized. Diagnostic and interventional endoscopy of small intestinal lesions remains a challenge due to anatomy. Endoscopy and radiological investigations, including magnetic resonance imaging and computerized tomography, are resourceful in the localization and biopsy of these lesions. For the small intestine, capsule endoscopy may be a particularly useful tool in the diagnostic process, however it does not afford the opportunity for 
real-time biopsy of any identified lesion.

Tissue examination demonstrates infiltrative vascular spaces lined by cells with significant cytologic atypia, characterized by hyperchromatic or vesicular nuclei with frequent layering and prominent nucleoli. A low-grade or well-differentiated AS may be mistaken for a hemangioma. However, recognition of an infiltrative growth pattern would be worrisome for a malignant neoplasm. A moderately differentiated AS may have a component of solid tumor growth and increased mitotic activity. A high-grade or poorly differentiated AS may be devoid of vasoformative regions, growing mostly in solid sheets or papillary pattern. The degree of vascular channel formation may be variable within different areas of the same tumor. Necrosis, hemorrhage and 'blood lakes,' hemosiderin, and mitoses are often seen but may be lacking in well-differentiated tumors. ${ }^{[4]}$ A poorly differentiated AS comprised of spindle-shaped cells may be a morphologic mimicker of a high-grade spindle cell sarcoma. Conversely, when comprised of epithelioid cells, poorly differentiated AS may easily be mistaken for a poorly differentiated carcinoma. $^{[4,16]}$ Hence, immunohistochemical markers play a vital role in the histologic diagnosis. Positive immunohistochemical markers include CD31, CD34, ERG, VEGFR2, and FLI-1. Keratin immunoreactivity can be seen in a subset of epithelioid AS, presenting a potential pitfall for a carcinoma misclassification. ${ }^{[17,18]}$ In a study of 8 cases of primary and metastatic intestinal AS, Allison et al. re- ported variable, focal to diffuse immunoreactivity with cytokeratin 7, Cam5.2/cytokeratin 8, cytokeratin 19, and cytokeratins AE1/AE3 immunohistochemical stains in all cases. However, all cases were non-reactive with cytokeratin 20 , distinguishing them from intestinal-derived carcinomas. ${ }^{[4]}$ Cytokeratin-positive carcinomas are negative for CD 31 and CD 34. Desmin, SMA, HHV-8, S100 protein, melanoma markers, and CAMTA-1 are usually negative in angiosarcomas. Absent immunoreactivity for the aforementioned markers helps to rule out the differential diagnostic considerations of leiomyosarcoma (desmin and SMA), Kaposi sarcoma (HHV-8), melanoma (S100 protein and melanoma markers), and epithelioid hemangioendothelioma (CAMTA$1)$.

In conclusion we are reporting a case of aggressive primary intestinal epithelioid AS that presented with intractable gastrointestinal bleeding. For epithelioid AS of the intestine, a tumor with aggressive features and poor prognosis, a high index of suspicion needs to be entertained in patients with persistent or recurrent gastrointestinal bleeding that is not responsive to endoscopic interventions or surgical resection. The use of immunohistochemical stains aids in the accurate diagnosis of this rare entity.

\section{CONFLiCTS OF INTEREST Disclosure}

No conflicts of interest exist for any of the authors included in this report.

\section{REFERENCES}

[1] Perez EA, Livingstone AS, Franceschi D, et al. Current incidence and outcome of gastrointestinal mesenchymal tumors including gastrointestinal stromal tumors. Journal of the American College of Surgeons. 2006; 202: 623-629. PMid:16571433. https://doi .org/10.101 6/j.jamcollsurg.2006.01.002

[2] Young RJ, Brown NJ, Reed MW, et al. Angiosarcoma. Lancet Oncology. 2010; 11: 983-991. PMid:20537949. https://doi.org/ $10.1016 / \mathrm{S} 1470-2045(10) 70023-1$

[3] Li R, Ouyang ZY, Xiao JB, et al. Clinical characteristics and prognostic factors of small intestine angiosarcoma: a retrospective clinical analysis of 66 cases. Cell Physiology and Biochemistry. 2017; 44: 817-827. PMid:30235449. https://doi.org/10.1159/000485 345

[4] Allison KH, Yoder BJ, Bronner MP, et al. Angiosarcoma involving the gastrointestinal tract: a series of primary and metastatic cases. American Journal of Surgical Pathology. 2004; 28: 298307. PMid:1504292. https://doi.org/10.1097/00000478-2 00403000-00002

[5] Nai Q, Ansari M, Liu J, et al. Primary small intestinal angiosarcoma: epidemiology, diagnosis and treatment. Journal of Clinical Medical Research. 2018; 10(4): 294-301. PMid:29511417. https://doi.org/10.14740/jocmr3153w
[6] Grewal JS, Daniel AR, Carson EJ, et al. Rapidly progressive metastatic multicentric epithelioid angiosarcoma of the small bowel: a case report and review of literature. International Journal of Colorectal Disease. 2008; 23(8): 745-756. PMid: 18080128. https: //doi.org/10.1007/s00384-007-0420-x

[7] Ni Q, Shang D, Peng H, et al. Primary angiosarcoma of the small intestine with metastasis to the liver: a case report and review of the literature. World Journal of Surgical Oncology. 2013; 11: 242. PMid: 24067058. https://doi.org/10.1186/1477-7819-11-242

[8] Mohammed A, Aliyu HO, Liman AA, et al. Angiosarcoma of the small intestine. Annals of African Medicine. 2011; 10(3): 246-248. https://doi.org/10.4103/1596-3519.84702

[9] Delvaux V, Sciot R, Neuville B, et al. Multifocal epithelioid angiosarcoma of the small intestine. Virchows Archiv. 2000; 437: 90-94. https://doi.org/10.1007/s004280000183

[10] Liu Z, Yu J, Xu Z, et al. Primary angiosarcoma of the small intestine metastatic to peritoneum with intestinal perforation: a case report and review of the literature. Translational Cancer Research. 2019; 8(4): 1635-1640. https://doi.org/10.21037/tcr.2019.06.40

[11] Ronen S, Ivan D, Torres-Cabala CA, et al. Post-radiation vascular lesions of the breast. Journal of Cutaneous Pathology. 2019; 46: 52-58.PMid:30251277. https://doi.org/10.1111/cup. 13363 
[12] Yu PC, Aplin B, Reed AB. Angiosarcoma of the abdominal aorta after endovascular aneurysm repair. Journal of Vascular Surgery Cases and Innovative Techniques. 2019; 5: 506-508. PMid:31763509. https://doi.org/10.1016/j.jvscit.2019.07.003

[13] Milite D, Pilan F, Ferrari A, et al. Aortic epithelioid angiosarcoma after endovascular aneurysm repair. Annals of Vascular Surgery. 2016; 35: 207.e17-21. https://doi.org/10.1016/j.avsg. 2016.02 .014

[14] Policarpio-Nicolas MCC, Nicolas MM, Keh P, et al. Postradiation angiosarcoma of the small intestine: a case report and review of literature. Annals of Diagnostic Pathology. 2006; 10(5): 301-305. PMid:16979525. https://doi.org/10.1016/j.anndiagpath . 2005.09.006

[15] Mesli SN, Ghouali AK, Benamara F, et al. Stewart-Treves syndrome involving chronic lymphedema after mastectomy of breast cancer. Case Reports in Surgery. 2017; 2017: 4056459. https: //doi.org/10.1155/2017/4056459

[16] Deshpande V, Rosenberg AE, O'Connell JX, et al. Epithelioid angiosarcoma of the bone: a series of 10 cases. Am J Surg Pathol. 2003; 27: 709-716. PMid:12766574. https://doi.org/10.109 7/00000478-200306000-00001

[17] Lin XY, Liu Y, Zhang Y, et al. The co-expression of cytokeratin and p63 in epithelioid angiosarcoma of the parotid gland: a diagnostic pitfall. Diagnostic Pathology. 2012; 7: 118. PMid:22943673. https://doi.org/10.1186/1746-1596-7-118

[18] Miettinen M, Fetsch JF. Distribution of keratins in normal endothelial cells and a spectrum of vascular tumors: implications in tumor diagnosis. Human Pathology. 2000; 31(9): 1062-1067. PMid:11014572. https://doi.org/10.1053/hupa.2000.9843 